\title{
Eliminating Redundant and Less-Informative RSS News Articles Based on Word Similarity and a Fuzzy Equivalence Relation
}

Ian Garcia

Yiu-Kai D. $\mathrm{Ng}$

ng@cs.byu.edu

Follow this and additional works at: https://scholarsarchive.byu.edu/facpub

Part of the Computer Sciences Commons

\section{Original Publication Citation}

Ian Gracia and Yiu-Kai Ng. "Eliminating Redundant and Less-Informative RSS News Articles Based on Word Similarity and A Fuzzy Equivalence Relation." In Proceedings of the 18th IEEE International Conference on Tools with Artificial Intelligence (ICTAI-26), pp. 465-473, November 13-15, 26, Washington, D.C.

\section{BYU ScholarsArchive Citation}

Garcia, lan and $\mathrm{Ng}$, Yiu-Kai D., "Eliminating Redundant and Less-Informative RSS News Articles Based on Word Similarity and a Fuzzy Equivalence Relation" (2006). Faculty Publications. 282.

https://scholarsarchive.byu.edu/facpub/282

This Peer-Reviewed Article is brought to you for free and open access by BYU ScholarsArchive. It has been accepted for inclusion in Faculty Publications by an authorized administrator of BYU ScholarsArchive. For more information, please contact ellen_amatangelo@byu.edu. 


\section{Eliminating Redundant and Less-Informative RSS News Articles Based on Word Similarity and a Fuzzy Equivalence Relation}

\author{
Ian Garcia \\ Computer Science Department \\ Brigham Young University, Provo, Utah
}

\author{
Yiu-Kai $\mathrm{Ng}$ \\ Computer Science Department \\ Brigham Young University, Provo, Utah
}

\begin{abstract}
The Internet has marked this era as the information age. There is no precedent in the amazing amount of information, especially network news, that can be accessed by Internet users these days. As a result, the problem of seeking information in online news articles is not the lack of them but being overwhelmed by them. This brings huge challenges in processing online news feeds, e.g., how to determine which news article is important, how to determine the quality of each news article, and how to filter irrelevant and redundant information. In this paper, we propose a method for filtering redundant and less-informative RSS news articles that solves the problem of excessive number of news feeds observed in RSS news aggregators. Our filtering approach measures similarity among RSS news entries by using the Fuzzy-Set Information Retrieval model and a fuzzy equivalent relation for computing word/sentence similarity to detect redundant and less-informative news articles.
\end{abstract}

\section{Introduction}

During the past decades, besides abundant amounts of information on the Web, the way information is released has also been changed. "Just a decade ago, large-scale flows of information, such as news feeds, were owned, monitored, and filtered by organizations specializing in the provision of news. The Web has brought the challenges and opportunities of managing and absorbing news feeds to all interested users" [4]. The traditional way in which Internet users access news is by visiting a Web site, and revisiting the Web site to check for updates on any information. Typical Internet users are not interested in the information of only one Web site, instead they often visit several Web sites, diversified for various sources of information. If an Internet user wants to remain current with updates posted by those Web sites, (s)he would have to visit all the selected Web sites several times a day, which is a tedious and inefficient process. Since accessing online news is one of the favorite activities of Internet users ${ }^{1}$, eliminating redundant and lessinformative online news articles could help Internet users save time in locating useful information and avoid the frustrating process of filtering replicated information.

In March 1999, Dan Libby created a method for syndicating information (originated by UserLand Software in 1997) for use on the My Netscape portal, called $\mathrm{RSS}^{2}$. RSS is a series of XML formatted files for Web syndication which has become the de-facto standard for news portals and has been widely adopted to release other type of information, such as Weblogs, commercial Web sites, job listings, bug reports, and government information. As it has been claimed in Feedster ${ }^{3}$, "Everything that is timely and valuable on the Web will be available as an RSS feed."

With the development of RSS, Internet users can (i) personalize the news they are interested in by including the headlines from the Web sites they access on a regular basis, and (ii) retrieve an RSS file containing not only the headlines, but a short description of each news and a link to the source of the news on one page, which is very useful. However, due to the large number of online news portals and huge amount of RSS news feeds, the user's challenge now is to process news articles in a timely manner. A possible solution to this problem is to deliver personalized news by removing redundant or less-informative news articles.

In this paper, we propose a filtering strategy for detecting and eliminating redundant and less-informative RSS news articles among the excessive number of news feeds entries. Our filtering approach measures similarity among RSS news entries by using the Fuzzy-Set Information Retrieval (IR) model and a word cluster for computing word/sentence similarity among news articles.

\footnotetext{
${ }^{1}$ According to the Newspaper Audience Database (NADbase), http:// www.naa.org/nadbase, one in three Internet users (55 million) visited a news portal over the course of a month, which was incremented $21 \%$ from the year of 2005 to the first quarter of the year of 2006.

${ }^{2}$ The abbreviation is used to refer to the following standards: Rich Site Summary (RSS 0.91), RDF Site Summary (RSS 0.9 and 1.0), and Really Simple Syndication (RSS 2.0).

${ }^{3}$ Feedster (http://www.feedster.com) is one of the first Web sites to search, crawl, and index Weblogs.
} 
We proceed to present our results as follows. In Section 2, we discuss related works in detecting similar documents. In Section 3, we introduce different word clusters and the Fuzzy-Set IR model for detecting similar and redundant RSS news articles. In Section 4, we present the detailed design on using a fuzzy equivalence relation for filtering less-informative RSS news articles. In Section 5, we give a concluding remark.

\section{Related Work in Similarity Measures}

Computing the similarity between documents has been extensively studied as an essential tool for applications such as text document searching [3], document clustering [16], copy or plagiarism detection $[9,12]$, text document retrieval, filtering, and categorization. Automatically determining whether two documents are similar and to what extent they are similar is a non-trivial problem.

The accuracy of similarity detection between two documents $d_{1}$ and $d_{2}$ relies heavily on computing the degree of similarity between $d_{1}$ and $d_{2}$, which can be determined by (i) the degree of lexical overlap in terms of the contents of $d_{1}$ and $d_{2}$, or (ii) the semantic contents, i.e., words/sentences, in $d_{1}$ and $d_{2}$. The semantic content approach goes beyond counting the number of words that appear in both $d_{1}$ and $d_{2}$, and the ability to assess the degree of semantic similarity between $d_{1}$ and $d_{2}$ automatically, scalably, and accurately is a key factor for justifying the effectiveness of information handling and decision support systems that detect similar text documents.

Many efforts have been made for computing the degree of similarity between two documents. From relatively simple programs, such as the diff command in UNIX/LINUX, which compares any two text documents in a line-by-line fashion and displays the contents and the line numbers where the two documents differ, through other more complex systems, such as COPS (COpy Protection System) [2], which is designed for detecting plagiarism. SIF [8] is one of the first copy-detection systems, which was intended not only for detecting similar text documents but binary documents as well. SIF, which considers the checksum of a file as its fingerprint, identifies similar files in a file system, and its approach is completely syntactic. COPS and SCAM (Stanford Copy Analysis Mechanism) [12] are two other copy-detection systems, which index a collection of documents by assigning hash values to sentences and paragraphs and comparing the hash values to determine the similarity among the corresponding documents. The main drawback of these copy detection approaches is the creation of a large number of collisions, same as other approaches that use hashing.

While SCAM is designed for comparing only small documents in a word-based fashion, document index graph (DIG) [5] is a document clustering system that uses a phrased-based matching model and an index model to detect similarity among documents. Even though Diff, SIF, $D I G, C O P S$, and SCAM are different systems with different design goals, they all adopt lexical comparison approaches and thus fail to consider lexically different but semantically the same or similar documents. We further enhance the semantic comparison method in [10, 14], called Fuzzy-Set Information Retrieval (IR) model, for detecting similar, but not necessary the same, documents.

\section{Word Clusters and the Fuzzy-Set IR Model}

Detecting redundant and less-informative RSS news articles is a challenging task, since RSS news feeds are dynamic in nature. The technology of RSS allows Internet users to subscribe to Web sites that typically add or modify content regularly and rapidly. To use this technology, site owners create or obtain specialized software (such as a content management system), which is in the machine-readable XML format, and present new articles in a list, including a line or two of each article and a link to the full article. (See, as an example of, an RSS news feed file as shown in Figure 1.) One of the essential elements in an RSS file is Channel, which contains several sub-elements that describe the information contained in the file. Sub-elements of Channel include (i) title, which is similar to the title tag in an HTML file and is used for identifying the RSS news feed. Usually, the content of title is the name of the Web site that provides the RSS file. (ii) Link, which is the URL of the Web site from where the RSS file can be retrieved. (iii) Description, which includes a sentence that briefly describes what the "stories," i.e., news articles, contained in the file are about, such as politics, economics, sports, etc. (iv) Item, which is a story identified by an $<$ item $>$ tag. Several items can be specified in the Channel element. The most important subelements of an item are (a) title, which contains the headline of the story, (b) link, which is the URL where the story in full can be retrieved, (c) description, which contains a few lines about the story and many times it is the first sentences of the story, and (d) pubDate, which is the date and time when the story is posted. We treat an item as a tuple of an RSS news feed, which contains the elements in the $<$ item $>$ tag, i.e., title, link, description, and pubDate.

We propose a selective filtering approach using the Fuzzy-Set IR model, where RSS news entries to be filtered are the ones that provide less-information or possess information which is already included in another RSS news entries, i.e., redundant, from either a different or the same RSS news feed. This can be achieved by comparing the RSS news entries and determining the similarity among them.

\subsection{The Fuzzy-Set IR Model}

The fuzzy set theory relies on two main concepts: (i) sets are not well-defined, and (ii) an element (e.g., a word) 


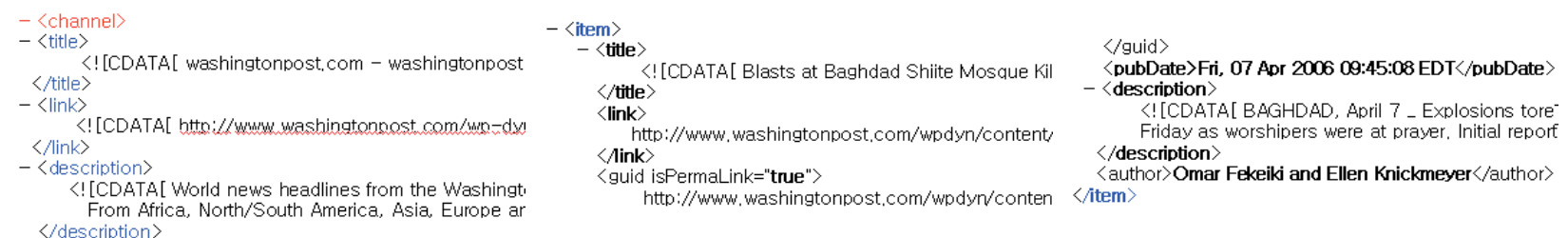

Figure 1. Portion of a RSS news feed file.

has a degree of membership to a set which falls within the range of the real interval $[0,1][6]$. In [14], the Fuzzy-Set IR model is adopted to determine whether a keyword in a sentence belongs to a (fuzzy) set of words, which have certain degrees of similarity among themselves. The degrees of similarity, refereed as correlation factors, among words are given by a function which assigns a value in the range $[0,1]$ to any two words. There are several methods to define the correlation factors among different words.

\subsection{The Correlation Factor}

The Fuzzy-Set IR model makes use of correlation factors, each of which is a similarity measure of any two words $w_{1}$ and $w_{2}$, i.e., the degree of similarity between $w_{1}$ and $w_{2}$. The correlation factor between $w_{i}$ and $w_{j}(i, j \geq 1)$ is given in a symmetric, correlation matrix $M$, where each entry $m_{i, j} \in M$ is the correlation factor between $w_{i}$ and $w_{j}$ (also called keywords in [1]) and $m_{i, j} \in[0,1]$.

In order to generate a correlation matrix of correlation factors, we must first collect a large number of "representative" English documents, which should be unbiased in terms of writing styles and diversed in contents, to calculate the correlation factors among distinct words according to their occurrences within each document of the collection. Some of the most popular sets of (archive) documents used in related projects are the TREC collection (http://trec.nist.gov/) and the Gutenberg project (http://www.gutenberg.org). These sets of documents, however, have major drawbacks. The Gutenberg project, which is a collection of books that is periodically augmented with new books, lacks a variety of topics, especially in science and technology. Even though the TREC collection includes a wide variety of topics, its public version has not been updated for several years. For these reasons, we have chosen the Wikipedia collection. Wikipedia [13] is a free online encyclopedia, which contains more than 930,000 articles and approximately 340 million words. The collection of Wikipedia articles, which are written by more than 89,000 volunteers, overcomes the drawbacks of the TREC and Gutenberg collections, since Wikipedia contains almost all possible topics in different areas of study, is constantly updated, and is unbiased in terms of writing styles and authorship. With the use of the Wikipedia documents to generate word-correlation factors, we can obtain a reliable sim- ilarity measures of different words according to their occurrences in various documents.

The Wikipedia articles are comprised in a single XML file of approximately $4.6 \mathrm{~Gb}$ in size. We first filtered the content in the "title" and "text" tags of each Wikipedia article. The filtering process requires two steps: (i) removing stopwords [1] and (ii) stemming remaining words [11]. Stopwords, which are words that are very common, such as prepositions and demonstrative, interrogative, and indefinite pronouns, do not provide useful information to distinguish the contents of different documents. Stopword removal is accomplished by verifying if a word is contained in the stopwords hash table, which is constructed by using several widely used stopword lists. Once the stopwords are removed, we proceed to stem all the remaining words using the Porter algorithm [11]. Quoting Martin Porter himself [11]: "The Porter stemming algorithm (or Porter stemmer) is a process for removing the commoner morphological and inflexional endings from words in English. Its main use is as part of a term normalization process that is usually done when setting up Information Retrieval systems." Hereafter, we processed the remaining non-stop, stemmed words ${ }^{4}$ and created a word-frequency-location file in which each record consists of (i) one of the words $w$, (ii) the document $D$ (identified by document number) where $w$ appears, (iii) the frequency of occurrence of $w$ in $D$, and all the positions where $w$ is present in $D$. (See Table 1 for portion of the word-frequency-location file.) The generated word-frequency-location file contains 144,048,788 records, and the number of non-stop, stemmed words in the file is 57,926 . The corresponding symmetric matrix contains $1,677,681,775=57,926 \times 57,925 / 2$ entries and takes up $6.3 \mathrm{~Gb}$ of disk space where each entry is stored as a 32-bit floating point number. Once the word-frequency-location file is generated, we can calculate a word-word correlation matrix by considering either (i) the number of documents (i.e., Wikipedia articles) in which two distinct terms $w_{1}$ and $w_{2}$ appear, which yields the keyword-connection correlation factors (matrix), (ii) the frequency of co-occurrence correlation factors of $w_{1}$ and $w_{2}$ in each document, which yields the co-occurrence matrix, and (iii) the distance correlation factors between $w_{1}$ and $w_{2}$ in each document, which yields the distance matrix.

\footnotetext{
${ }^{4}$ From now on, unless stated otherwise, whenever we use the term "word," we really mean "non-stop, stemmed word."
} 


\begin{tabular}{cccl}
\hline \hline Keyword & Doc $(D)$ & Frequency & Positions in $D$ \\
\hline computer & 5 & 5 & $3,21,40,32,88$ \\
computer & 12 & 3 & $14,45,100$ \\
comrade & 1 & 2 & 30,64 \\
comrade & 21 & 3 & $8,30,58$
\end{tabular}

Table 1. Some keyword Frequencies and Positions in the word-frequency-location file

\subsubsection{Keyword Connection}

The (key)word-connection correlation factor, which has been used for comparing similarity among documents, calculates the correlation of any two words $w_{1}$ and $w_{2}$ by counting the number of documents in a collection $C$ where both $w_{1}$ and $w_{2}$ appear together $[1,10]$. In the keywordconnection matrix [10], each entry $m_{i, j}$, i.e., the correlation factor, for $w_{i}$ and $w_{j}$ is calculated as

$$
c_{i, j}=\frac{n_{i, l}}{n_{i}+n_{l}-n_{i, l}}
$$

where $n_{i}\left(n_{l}\right.$, respectively) is the number of documents in $C$ in which the keyword $w_{i}\left(w_{l}\right.$, respectively) appears, and $n_{i, l}$ is the number of documents in $C$ in where $w_{i}$ and $w_{l}$ appear. The correlation factors of different keywords computed by using the keyword-connection method follow the conjuncture that "The more documents in which two keywords occur, the more they relate to each other." [10]

The keyword-connection matrix is simpler to compute than the co-occurrence and the distance matrices; however, a major drawback of this simplicity is accuracy, since keyword-connection correlation factors do not consider other factors among different words, which include (i) the frequency of co-occurrence of any two words within a document and (ii) how close any two words appear together in a document. These factors are further considered by the co-occurrence frequency matrix (i.e., association cluster in [1]) and the distance matrix (i.e., metric cluster in [1]), respectively in computing the correlation factors of any two words.

\subsubsection{Co-Occurrence Frequency}

The co-occurrence correlation factor not only considers the number of documents in a collection where both words $w_{1}$ and $w_{2}$ appear, but it also considers the frequency of cooccurrence of both $w_{1}$ and $w_{2}$ in a document. In order to compute the co-occurrence factors, we obtain a frequency matrix $m$ where each entry $f_{i, d_{u}}$ is the frequency of word $i$ in document $d_{u}$. The composition of $m$ and its transpose, i.e., $m^{t}$, yields the matrix $c=m \cdot m^{t}$, where each entry $c_{i, j}$ of the matrix is the co-occurrence frequency factor of words $w_{i}$ and $w_{j}$, i.e.,

$$
c_{i, j}=\sum_{n=1}^{l}\left(f_{i, d_{n}} \times f_{j, d_{n}}\right)
$$

where $l$ is the total number of documents in a collection. We can normalize each correlation factor to limit the values in the interval $[0,1]$ as

$$
c_{i, j_{\text {norm }}}=\frac{c_{i, j}}{c_{i, i}+c_{j, j}-c_{i, j}}
$$

The frequency of co-occurrence of any two words in a document yields a more accurate correlation factor of the words than using the keyword-connection approach. Consider in an Wikipedia article about Shakespeare (http://en.wikipedia.org/wiki/Shakespeare) in which Shakespeare appears sixty times, english appears twenty times, and french appears only one time. If we use the keyword-connection method, the correlation factor of Shakespeare and english will be the same as the correlation factor between Shakespeare and french. However, it is clear that the correlation factor between Shakespeare and english should be higher.

Even though the co-occurrence frequency matrix considers the co-occurrence frequency of any two words, it does not consider how close any two words are as they appear in a document, which is another important factor in providing an accurate correlation factor of any two words.

\subsubsection{The Distance Correlation}

The distance correlation factor between any two words $w_{1}$ and $w_{2}$ considers the frequency of occurrence, as well as the "distance," that is measured by the number of words, between $w_{1}$ and $w_{2}$ within a document as an additional factor to calculate the correlation factor of $w_{1}$ and $w_{2}$. In the distance correlation approach, it is assumed that keywords which appear closer together are more likely related than those that appear far apart in the same document. For example, in a document that discusses computer architecture, the two words "computer" and "architecture" are likely to appear closely together most of the times, and their correlation factor computed by using their distances would be higher if their positions are considered, along with their frequency of co-occurrence. However, if a document discusses the usage of computers by architects for creating the blue prints of home designs, the keyword-connection or co-occurrence correlation factor, could give the same correlation factor to "computer" and "architecture" in both documents, which is inaccurate, since it is clear that in the document talking about computer architecture, the correlation factor is higher between the two words, whereas in the document about architects using computers, the correlation factor of the same two words should be smaller. 
The distance $d\left(w_{i}, w_{j}\right)$ between any two words $w_{i}$ and $w_{j}$ is defined by the (absolute) difference of the positions of any occurrence of $w_{i}$ and $w_{j}$ in a document, i.e., $d\left(w_{i}, w_{j}\right)=\left|\operatorname{Position}\left(w_{i}\right)-\operatorname{Position}\left(w_{j}\right)\right|$, and $d\left(w_{i}, w_{j}\right)=\infty$ when $w_{i}$ and $w_{j}$ do not appear in the same document. For each document $d_{u}$ in a collection $C$, the distance among each occurrence of $w_{i}$ and each occurrence of $w_{j}$ in $d_{u}$ is calculated, and the correlation factor of $w_{i}$ and $w_{j}$, i.e., $c_{i, j}$, is computed as the sum of the inverse of the distances between any occurrence of $w_{i}$ and $w_{j}$ :

$$
c_{i, j}=\sum_{w_{i} \in V\left(s_{i}\right)} \sum_{w_{j} \in V\left(s_{j}\right)} \frac{1}{d\left(w_{i}, w_{j}\right)}
$$

where $V\left(S_{i}\right)\left(V\left(S_{j}\right)\right.$, respectively), denotes the sets of words that include $w_{i}\left(w_{j}\right.$, respectively) and its respective stemmed words. We normalize the distance correlation factors in the interval $[0,1]$, as in the other correlation matrices, to redefined $c_{i, j}$ as

$$
s_{i, j}=\frac{c_{i, j}}{\left|V\left(S_{i}\right)\right| \times\left|V\left(S_{j}\right)\right|}
$$

Event though the calculation of the distance matrix is more complex than the keyword-connection and the cooccurrence matrices, the distance matrix is computed only once and captures the correlation factors of two words $w_{i}$ and $w_{j}$ more accurately than the other two, which will be verified in Section 3.3. We adopt the distance matrix approach in measuring the degrees of similarity among different words, which is used in the Fuzzy-Set IR model, for detecting redundant or less-informative RSS news entries.

\subsection{Word-Sentence-Document Fuzzy As- sociation}

Once a word-to-word correlation matrix is calculated, we can define a fuzzy set association of each word and a sentence, paragraph, or document itself. Since a news article in RSS news feeds includes only a brief summary, i.e., the 3-4 line summary, in the Title and Description sections, we treat the Title and Description sections as the content of an RSS news article such that the degree of similarity between any two RSS news articles is determined by the correlation factors among the words in the respective Title and Description sections of the two articles 5 .

The association between a keyword $k_{i}$ and a document $d_{j}$ (i.e., an RSS news article in this paper), referred in [10] as the word-document correlation factor $\mu_{i, j}$, is calculated as the complement of a negated algebraic product of all the

\footnotetext{
${ }^{5}$ If the actual contents of two RSS news articles $n_{1}$ and $n_{2}$ are used instead of the summaries of $n_{1}$ and $n_{2}$, the accuracy of the degree of similarity of $n_{1}$ and $n_{2}$ could be further enhanced, assuming that the processing time is not a main concern.
}

correlations of the (key)word $k_{i}$ and each (key)word $k_{l} \in$ $d_{j}$, i.e.,

$$
\mu_{i, j}=1-\prod_{w_{k} \in d_{j}}\left(1-c_{i, k}\right)
$$

The correlation value $\mu_{i, j}$ falls in the interval $[0,1]$ and reaches its maximum when $c_{i, k}=1$, for any $k \in d_{j}$. In [14], the $\mu_{i, j}$ factor is modified to compute the wordsentence correlation factor between word $i$ and sentence $j$, instead of computing the word-document correlation factor as defined in [10]. In this paper, we adopt the modification, i.e., $\mu_{i, j}$, as the correlation factor between keyword $k_{i}$ and sentence $S_{j}$. Since each RSS news article contains at most three short sentences (on the average in its RSS file as explained earlier), we treat them as a single sentence.

The degree of similarity of sentence $S_{i}$ (in an RSS news article) with respect to sentence $S_{j}$ (in another RSS news article), denoted as $\mathrm{Sim}_{i, j}$, is calculated as the average of all the values $\mu_{i, j}$, where $w_{l} \in S_{i}(1 \leq l \leq n)$, with respect to (all the words in) $S_{j}$ as

$$
\operatorname{Sim}_{i, j}=\frac{\mu_{w_{1}, j}+\mu_{w_{2}, j}+\cdots+\mu_{w_{n}, j}}{n}
$$

and $\operatorname{Sim}_{i, j} \in[0,1]$. It is important to note that in general, $\operatorname{Sim}_{i, j} \neq \operatorname{Sim}_{j, i}$. When $\operatorname{Sim}_{i, j}=0$, it indicates that there are no words in sentence $S_{i}$ that can be considered similar to any word in sentence $S_{j}$. If $\operatorname{Sim}_{i, j}=1$, then either sentence $S_{i}$ is (semantically) identical to sentence $S_{j}$, or $S_{i}$ is subsumed by sentence $S_{j}$, i.e., all the words in $S_{i}$ are (semantically) the same as (some of) the words in $S_{j}$.

An $E Q$ function of $\operatorname{Sim}_{i, j}$ and $\operatorname{Sim}_{j, i}$ is defined below, which determines whether sentences $S_{i}$ and $S_{j}$ should be considered as (semantically) the same.

$$
E Q\left(S_{i}, S_{j}\right)=\left\{\begin{array}{cc}
1 & \text { if } \operatorname{Min}\left(\operatorname{Sim}_{i, j}, \operatorname{Sim}_{j, i}\right) \geq \alpha \\
& \wedge\left|\operatorname{Sim}_{i, j}-\operatorname{Sim}_{j, i}\right| \leq \epsilon \\
0 & \text { otherwise. }
\end{array}\right.
$$

The parameter $\alpha$ is called the permission threshold value, and it determines the minimum similarity value for which $S_{i}$ and $S_{j}$ can be considered to be (semantically) the same. The second parameter $\epsilon$ is called the variation threshold value, which defines the maximum difference (in terms of the degree of similarity), between $S_{i}$ and $S_{j}$, for which $S_{i}$ and $S_{j}$ can be considered to be (semantically) the same. The values for $\alpha$ and $\epsilon$ were empirically obtained using a data set consisting of twenty-six RSS news articles retrieved from different RSS news feeds, which include Associated Press (http://www.ap.com), Yahoo (http://news.yahoo.com), and Boston Globe (http://www.bostonglobe.com). The number of possible combinations of the matching news articles pairs is $26 \times 25 \div 2=325$. (We manually evaluated them and counted a total of 17 matching pairs among all the news articles.) We considered the word-correlation factors in each 


\begin{tabular}{lcccc}
\hline \hline Correlation Matrix & FP & FN & M & Accuracy \% \\
\hline Keyword-connection & 2 & 9 & 8 & 47 \\
Co-ocurrance & 1 & 8 & 9 & 52 \\
Distance & 3 & 1 & 16 & $\mathbf{9 4}$ \\
\hline
\end{tabular}

FP: F(alse)P(ositive); FN: F(alse)N(egative); M(atches)

\section{Table 2. Experimental Results on twenty-six RSS news using the three different matrices}

of the different matrices, i.e., the keyword-connection, cooccurrence, and distance matrices, that were previously created using the Wikipedia articles and tested for the degrees of similarity among the twenty-six articles. We counted (i) the number of pairs of RSS news entries that were considered redundant or less-informative when in fact they are not (i.e. false positives) and (ii) the number of pairs that were considered different but are in fact redundant or lessinformative (i.e., false negatives).

The results we obtained by using each of the three matrices are shown in Table 2, which demonstrates that the correlation factors in the distance matrix provide the most accurate results among all the three matrices. These experimental results confirm the correct choice of the distance matrix and the $E Q$ function for detecting redundant or lessinformative news articles.

\section{Fuzzy Equivalence Relation}

By adopting the Fuzzy-Set IR model and the distance matrix, we can discard redundant RSS news articles, i.e., articles that do not provide new information. This task can be accomplished by eliminating any RSS news article $s$ such that $\operatorname{Sim}_{s, t}=1$, where $t$ is another RSS news article, which implies that the information contained in $s$ is entirely subsumed by $t$. Hereafter, we proceed to eliminate less-informative RSS news articles. This task can be accomplished by first generating clusters of all the RSS news articles that maintain certain degree of similarity. Note that news articles in the same cluster should be closely related, whereas distinct news articles belonged to different clusters should be significantly different in terms of their degrees of similarity. By deleting news articles in a non-singleton cluster $C$, which have the highest degree of similarity with respect to other news articles in $C$, we can eliminate lessinformative news articles.

Clusters of news articles can be created by adopting a fuzzy equivalence relation that applies to the news articles to generate "crisp" subsets (i.e., clusters). News articles in each cluster $C$ that are "less-informative" than others in $C$ are discarded. A cluster of RSS news articles is defined as $C_{\alpha}=\left\{d \mid S_{S_{i, e}} \geq \alpha, \forall e \in C_{\alpha}\right\}$, where $\alpha$ is the minimum degree of similarity such that any two news articles in $C_{\alpha}$ must hold. We generate clusters of non-redundant RSS news articles collected from various RSS news feeds by considering a fuzzy equivalence relation as given in [6]. A fuzzy equivalence relation defines a "crisp" equivalence relation among the elements of a set, which have been widely studied to measure the degree of similarity among different elements in a set. A (similarity) relation $R$ is a fuzzy equivalence relation if it is reflexive, symmetric, and max-min transitive, i.e.,

$$
\begin{gathered}
R(x, x)=1, \quad \forall x \in R \\
R(x, y)=R(y, x), \quad \forall x, y \in R \\
R(x, z) \geq \max _{y \in Y} \min \{R(x, y), R(y, z)\}
\end{gathered}
$$

where $Y$ is a fuzzy set and $x, y, z \in Y$.

The key for establishing a fuzzy equivalence relation is the definition of transitivity. The first definition for fuzzy transitivity was proposed by Zadeh [15], which is called the max-min transitivity, as defined in Equation 11. However, the max-min transitivity is known to be a restrictive constraint, which is not applicable to the similarity relation problem that we deal with. This is because in order to apply the max-min transitivity to our similarity problem, it is required that for any two news articles (i.e., documents) $d_{x}$ and $d_{z}$, there cannot exist another article $d_{y}$ whose similarities with both $d_{x}$ and $d_{z}$ is greater than the similarity between $d_{x}$ and $d_{z}$, i.e., the relation $R$ is not max-min transitive if given $d_{x}$ and $d_{z}$, there exists $d_{y}$ such that $R\left(d_{x}, d_{z}\right)<R\left(d_{x}, d_{y}\right)$ and $R\left(d_{x}, d_{z}\right)<R\left(d_{y}, d_{z}\right)$. Consider an example with the following sentences and the degrees of similarity $\operatorname{Sim}_{1,2}=0.20, \operatorname{Sim}_{1,3}=0.80, \operatorname{Sim}_{2,1}$ $=0.33, \operatorname{Sim}_{2,3}=1.00, \operatorname{Sim}_{3,1}=0.50$, and $\operatorname{Sim}_{3,2}=0.37$ :

\section{$S_{1}$ : Bush's proposal will benefit illegal immigrants.}

$S_{2}$ : The war in Iraq is not over said Bush.

\section{$S_{3}$ : Bush proposed a bill that will benefit immigrants and he said the war in Iraq will continue.}

In order to establish a fuzzy equivalence relation that satisfies the max-min transitivity for $S_{1}, S_{2}$, and $S_{3}$, it is necessary to define a relation (function) $R$ of the similarity values among $S_{1}, S_{2}$, and $S_{3}$ such that

$$
\begin{aligned}
& f(0.20,0.33) \geq \min \{f(0.80,0.50), f(1.0,0.37)\} \\
& f(0.80,0.50) \geq \min \{f(0.20,0.33), f(1.0,0.37)\} \\
& f(1.00,0.37) \geq \min \{f(0.80,0.50), f(0.33,0.2)\}
\end{aligned}
$$

We consider a function that takes two input values, which are the similarity measures between two documents $i$ and $j$ and returns a high value if the two input values are high. The function $f$ as shown in Equations 12, 13, and 14 does not satisfy this criteria, since $f$ in each of the inequality equations yields a high value when the given values are low. 
There exists another fuzzy transitivity relation, the $\max$ prod transitivity [6] (as defined below), which can be adopted to establish a fuzzy equivalence relation.

$$
R(x, z) \geq \max _{y \in Y}\{R(x, y) \times R(y, z)\}
$$

where $x, y, z$, and $Y$ are as defined in Equation 11 .

The max-prod transitivity is not as restrictive as the max-min transitivity and can be more easily satisfied by a function $R$ whose values fall in the interval $[0,1]$, since the product of two numbers $x, y \in[0,1]$ in the max-prod transitivity is smaller than $x$ and $y$, i.e., if $x, y \in[0,1]$, then $x \geq x \times y$ and $y \geq x \times y$. For this reason, we consider the max-prod transitivity instead of the max-min transitivity.

In order to adopt the fuzzy equivalence relation with max-prod transitivity for eliminating less-informative RSS news articles, it is necessary to define a function that combines the similarity measures of two news articles $d_{i}$ and $d_{j}$, i.e., $S i m_{i, j}$, and $S i m_{j, i}$, into a single one. We consider several functions and choose $Q$ in Equation 16 that satisfies the max-prod transitivity property as the desired function.

\subsection{Combination Functions}

One of the most commonly used combination equations is average. However, the average of two pairs of different similarity values, e.g., $(0.5,0.5)$ and $(0.9,0.1)$, can yield the same result, e.g., $(0.5+0.5) / 2=(0.9+0.1) / 2$. In addition, the average function is not max-prod transitive.

In [14], an $E Q\left(S_{i}, S_{j}\right)$ function of the similarity values of two documents $d_{i}$ and $d_{j}$, i.e., $\operatorname{Sim}_{i, j}$ and $S i m_{j, i}$, is defined (as given in Equation 8), which determines whether $d_{i}$ and $d_{j}$ should be considered as the same. The $E Q$ function, which is a discrete function, assigns the values 1 or 0 , and is symmetric and reflexive; however, $E Q$ is neither $\max$ min transitive nor max-prod transitive. Furthermore, one of the drawbacks of $E Q$ is that its two estimated threshold parameter values, i.e., $\alpha$ and $\epsilon$, must be established before $E Q$ can be adopted.

In [7], two combination equations that combine two values, e.g., $\operatorname{Sim}_{i, j}$ and $\mathrm{Sim}_{j, i}$, are defined as follows:

$$
\begin{array}{r}
Q\left(\operatorname{Sim}_{i, j}, \operatorname{Sim}_{j, i}\right)=\frac{\operatorname{Sim}_{i, j}+\operatorname{Sim}_{j, i}}{1-\min \left(\operatorname{Sim}_{i, j}, \operatorname{Sim}_{j, i}\right)} \\
Q_{1}\left(\operatorname{Sim}_{i, j}, \operatorname{Sim}_{j, i}\right)=\left(\operatorname{Sim}_{i, j}+\operatorname{Sim}_{j, i}\right)- \\
\left(\operatorname{Sim}_{i, j} \times \operatorname{Sim}_{j, i}\right)
\end{array}
$$

Both functions $Q$ and $Q_{1}$ are simple to compute; however, $Q_{1}$ has the similar drawback as the average function, i.e., it yields the same result to different pairs of values. For example, both (similarity) value pairs $(0.9,0.9)$ and $(0.99$, 0.1 ) are assigned the same value 0.99 by $Q_{1}$. In contrast, $Q$ assigns a high value only when both similarity measures of two documents $d_{i}$ and $d_{j}$ are high. Furthermore, function $Q$ in Equation 16 is fuzzy-symmetric, however, $Q$ is neither fuzzy-reflexive nor fuzzy-transitive. We modify $Q$ so that the modified $Q$ function, i.e., $E$ (given below), is a fuzzy equivalence relation.

$$
E\left(d_{i}, d_{j}\right)= \begin{cases}1 & \text { if } i=j \\ 0.0001 & \text { if } Q\left(d_{i}, d_{j}\right)<0.0001 \\ \frac{Q(X, Y)}{\max (Q(W, Z))} & \text { otherwise }\end{cases}
$$

where $X$ and $Y$ are $S i m_{i, j}$ and $S i m_{j, i}$, respectively, and $W$ and $Z$ are $S i m_{l, k}$ and $S i m_{k, l}$, respectively for any $d_{k}$ and $d_{l}$ in the set of documents which includes $d_{i}$ and $d_{j}$.

The first condition in Equation 18 is introduced to satisfy reflexivity, whereas the third condition is the normalized $Q$ function, which restricts the values of $E$ to the interval [0, $1]$. The second condition guarantees max-prod transitivity.

We have experimentally verified that the $E$ function is max-prod transitive, using twelve sets of news articles $S$ from different RSS news feeds and evaluating the maxprod transitivity inequality (as given in Equation 15) for each set of news articles. We consider every possible pair of news articles $d_{x}$ and $d_{z}$ in each RSS news feed and compute every possible $E\left(d_{x}, d_{y}\right) \times E\left(d_{y}, d_{z}\right)$ value to verify that $E\left(d_{x}, d_{z}\right) \geq E\left(d_{x}, d_{y}\right) \times E\left(d_{y}, d_{z}\right)$, for every $d_{y}$. The paramenter value 0.0001 , which was obtained empirically using $S$, limits the similarity value between any two news articles so that the max-prod transitivity is satisfied. It is essential to understand that the value 0.0001 is not simply set up to satisfy the max-prod transitivity. In fact, given any two documents $d_{1}$ and $d_{2}$ that are similar, if there exists another document $d_{3}$ such that $d_{1}$ and $d_{3}$, as well as $d_{3}$ and $d_{2}$, are similar, then the max-prod transitivity using 0.0001 always holds for $d_{1}, d_{2}$, and $d_{3}$.

\subsection{Clustering and Discarding Less- Informative News Articles}

After the fuzzy equivalence relation $E$ is established, we can apply $E$ to determine the equivalence classes (clusters) of news articles from different RSS news feeds by setting an $\alpha$-cut value [6]. As the value of $\alpha$ increases, the number of equivalence classes of the $\alpha$-cut also increases, whereas the size of each equivalence class is reduced. An $\alpha$-cut value guarantees that every pair of news articles in the same cluster has the degree of similarity at least equal to $\alpha$. We discard one or more (but not all) news articles from each cluster. We do not consider clusters with only one article, since they include the only news article which is dissimilar to other news articles in other clusters, assuming that our equivalence class generation approach is correct.

Since the same news article may appear in different clusters $^{6}$, we cannot consider each cluster separately while se-

\footnotetext{
${ }^{6} \mathrm{~A}$ fuzzy equivalence relation based on the max-prod transitivity does
} 
lecting less-informative news articles to discard. Instead, we rank the news articles in different clusters generated by an $\alpha$-cut value and discard those that have higher rankings, i.e., articles that are highly similar to others and thus are "less-informative," in the same cluster. While discarding news articles from different clusters, we cannot rank the news articles simply based on their similarity values, since we could discard all the news articles in a cluster. Consider the set of news articles $C=\{a, b, c, d, e, f\}$ and their similarity values: $\operatorname{Sim}_{a, b}=0.8, \operatorname{Sim}_{b, a}=0.85, \operatorname{Sim}_{c, d}=0.6$, $\operatorname{Sim}_{d, c}=0.7, \operatorname{Sim}_{e, f}=0.61$, and $\operatorname{Sim}_{f, e}=0.5$, whereas all the other similarity values are 0.2 , except the similarity value of the same pair of documents, which is 1.0. Using Equation 18, the $E$ value of each pair of news articles can be computed by using their similarity values, i.e., $E(a, b)$ $=0.055, E(c, d)=0.021, E(e, f)=0.014$, whereas all the other possible pairs yield the same $E$ value, i.e., 0.0025 . If we set $\alpha=0.1$, then three clusters $C_{1}=\{a, b\}, C_{2}=\{c, d\}$, and $C_{3}=\{e, f\}$ are generated. Suppose we need to discard two news articles. If we simply discard the top two articles with the highest similarity values, then we would discard $a$ and $b$ in $C_{1}$, which is an undesirable result.

\subsection{Different Ranking Approaches}

We compare three different approaches in ranking the news articles in each cluster generated by an $\alpha$-cut value. In the first approach, we consider the entropy value of each news article. The entropy in information theory is an alternative way to describe how much information is carried in a document and thus can be used to determine the relative degrees of similarity among different news articles. We measure the entropy of each news article by using

$$
H(x)=-\sum_{i=1}^{N} p(i) \log _{2} p(i)
$$

where $x$ represents a non-redundant news article and $N$ is the number of clusters to where $x$ belongs.

We have chosen the $Q$ function in Equation 16 to compute probability $p$ in Equation 19 for each news article. Table 3 shows the ranking according to the entropy values of a set of news articles (collected from various RSS news feeds) in different clusters generated by an $\alpha$-cut value. By using the number of occurrences of each news article in different clusters, we realize that there is a clear bias towards those articles that have higher frequency of occurrences, which is not a desired behavior, since news articles that appear several times in different clusters are not necessarily the ones that have the higher degree of similarity with a particular news article than the remaining news articles.

not always yield disjoint equivalence classes, which is different from the fuzzy equivalence relation based on the $\max$-min transitivity.

\begin{tabular}{|r|c|c||r|c|c||c|c|c|}
\hline \multicolumn{3}{|c||}{ Average $M$} & \multicolumn{3}{c|}{ Entropy $(H)$} & \multicolumn{3}{c|}{ Average $E$} \\
\hline Art. & F & Rank & Art. & F & Rank & Art. & F & Rank \\
\hline 204 & 1 & 0.93 & 36 & 4 & 0.27 & 222 & 1 & 0.06 \\
41 & 1 & 0.93 & 67 & 3 & 0.24 & 218 & 1 & 0.06 \\
299 & 2 & 0.87 & 137 & 1 & 0.15 & 204 & 1 & 0.02 \\
116 & 1 & 0.86 & 299 & 2 & 0.13 & 324 & 1 & 0.02 \\
36 & 4 & 0.76 & 89 & 2 & 0.10 & 89 & 2 & 0.01 \\
271 & 3 & 0.72 & 324 & 1 & 0.10 & 49 & 1 & 0.01 \\
67 & 3 & 0.71 & 415 & 1 & 0.09 & 299 & 2 & 0.01 \\
$\ldots$ & $\ldots$ & $\ldots$ & $\ldots$ & $\ldots$ & $\ldots$ & $\ldots$ & $\ldots$ & $\ldots$ \\
\hline
\end{tabular}

Art(icle): identified by its article number; F(requency)

\section{Table 3. Article rankings using Equations 20, 19 , and 18.}

We have considered another ranking, which is the average of the $E$ values (in Equation 18) of news articles. The average includes all the $E$ values obtained between a document $d_{i}$ and each of the other documents $d_{j}$ that appear in each of the clusters to where $d_{i}$ belongs. However, the average of the $E$ values does not yield a good ranking either, since the average of the $E$ values assigns a low ranking to articles 41 and 36. Article 41 should have a high ranking and be discarded, since it is very similar to article 28 , and article 36 should also receive a high ranking, since (i) it appears in several clusters and (ii) has high similarity values with those news articles in the clusters. Instead of adopting the entropy or the average $E$ values, we use the function $M$ in Equation 20 to rank news articles in all clusters that include at least two news articles generated by an $\alpha$-cut value. $M$ provides the average of the maximum similarity values of a news article $d_{i}$ with another news article $d_{j}$ in each cluster where $d_{i}$ appears.

$$
M\left(d_{i}\right)=\frac{\sum_{k=1}^{N} \max _{C_{k}}\left\{\operatorname{Sim}_{i, j}\right\}}{N}
$$

The ranking based on the average of $H$ values considers the trade off between the (i) the frequency of a news article in clusters generated by an $\alpha$-cut value and (ii) the similarity values of a news article with respect to other news articles in various clusters, which yields the most accurate ranking on news articles according to the conducted experiments. According to the rankings, the top $n(n \geq 1)$ ranked (i.e., less-informative) news articles, along with all the redundant ones that have been detected earlier, are discarded, assuming that their frequencies of occurrence is greater than 1 , i.e., they occur in non-singleton clusters.

Example 1 Consider a set of ten RSS news articles that were extracted from various RSS news feeds as shown in Table 4 and the non-singleton clusters that were generated as shown in Figure 2. The clusters and the computed rankings of the ten articles can be used to determine which one(s) of the ten articles should be discarded. 


\begin{tabular}{|l|l|}
\hline ID & Title and URL \\
\hline \hline$a_{0}$ & House Considers Bill to Boost Refineries. abcnews.go.com/Business/wireStory?id=1191725\&amp \\
\hline$a_{1}$ & Intruder Gains Front Lawn of White House. abcnews.go.com/Politics/wireStory?id=1823761 \\
\hline$a_{2}$ & Screaming Intruder Jumps White House Fence. www.examiner.com/a-73142 \\
\hline$a_{3}$ & Saudi Ambassador: Iraq Invasion Helped Spread Terrorism. www.foxnews.com/story/0,2933,178188,00.html \\
\hline$a_{4}$ & Bush says Iraqi parliamentary elections “a major step forward". english.people.com.cn/200512/16/eng20051216 \\
\hline$a_{5}$ & Powerball Winners Donate to Homeless. www.foxnews.com/story/0,2933,190670,00.html \\
\hline$a_{6}$ & mSleep disorders affect millions of Americans. english.people.com.cn/200604/05/eng20060405_256141.html \\
\hline$a_{7}$ & Millions of Americans suffer sleep disorders. news.xinhuanet.com/english/2006-04/05/content_4386829.htm \\
\hline$a_{8}$ & Gasoline price drop may only be temporary. www.boston.com/news/nation/washington/articles/2005/09/19/ \\
\hline$a_{9}$ & House considers bill to boost refineries. abcnews.go.com/Business/wireStory?id=1191983\&page=1 \\
\hline
\end{tabular}

Table 4. Ten RSS news articles downloaded from various RSS news feeds and their URLs

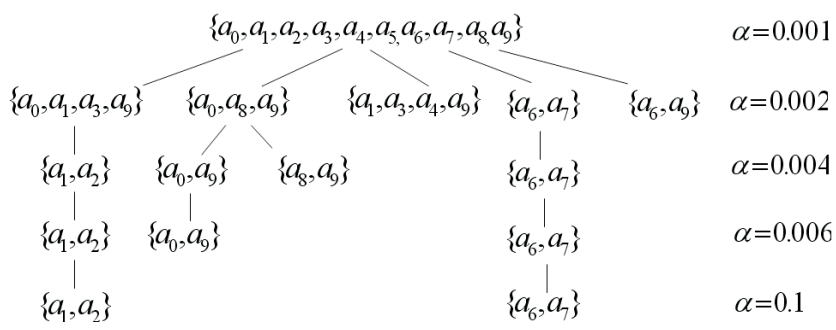

Figure 2. Clusters generated on the set of 10
RSS news articles as shown in Table 4 ac-
cording to different $\alpha$-cut values

The number of "less-informative" news articles to be discarded can be determined by using (i) the average number of new articles that are accessed by an individual user, and (ii) the number of (new or updated) articles posted by each individual RSS news feed that the user accesses on a regular basis.

\section{Conclusions}

In this paper, we propose a solution to the RSS news information overflow problem by providing a filtering approach which selectively eliminates redundant or lessinformative RSS news feeds entries. The proposed filtering approach, which adopts the Fuzzy-Set IR model, a distance matrix, and a fuzzy equivalence relation with maxprod transitivity, can detect redundant and less-informative RSS news articles accurately. Furthermore, we have fully implemented our filtering approach in PERL and JAVA.

For future work, we would like to investigate using natural language processing, user personal profiles, and unsupervised machine learning approaches to further enhance our filtering method by taking into consideration the user preference and other semantic constraints detected from source documents.

\section{References}

[1] R. Baeza-Yates and B. Ribeiro-Neto. Modern Information Retrieval. Addison Wesley, 1999.

[2] S. Brin, J. Davis, and H. Garcia-Molina. Copy detection mechanisms for digital documents. In Processings of ACM SIGMOD, pages 398-409, 1995.

[3] S. Chien and N. Immorlica. Semantic similarity between search engine queries using temporal correlation. In Processings of the World Wide Web Conf., pages 2-11, 2005.

[4] E. Gabrilovich, S. Dumais, and E. Horvitz. Newsjunkie: Providing personalized newsfeeds via analysis of information novelty. In Processings of the World Wide Web Conference, pages 482-490, 2004.

[5] K. Hammouda and M. Kamel. Efficient phrase-based document indexing for web document clustering. IEEE TKDE, 16(10):1279-1296, 2004.

[6] G. Klir, U. St. Clair, and B. Yuan. Fuzzy Set Theory, Foundations and Applications. Prentice Hall, 1997.

[7] G. Luger. Artificial Intelligence, Structures and Strategies for Complex Problem Solving. Addison Wesley, 1997.

[8] U. Manber. Finding similar files in large file system. In Processings of the USENIX Winter Technical Conf., 1994.

[9] H. Nevin. Scalable document fingerprinting. In Processings of the $2^{\text {nd }}$ USENIX Workshop on Electronic Commerce, pages 191-200, 1996.

[10] Y. Ogawa, T. Morita, and K. Kobayashi. A fuzzy document retrieval system using the keyword connection matrix and a learning method. Fuzzy Sets \& Systems, 39:163-179, 1991.

[11] M. Porter. An algorithm for suffix stripping. Program, 14(3):130-137, 1980.

[12] N. Shivakumar and H. Garcia-Molina. The scam approach to copy detection in digital libraries. Diglib Magazine, 1995.

[13] Wikipedia. http://wikipedia.org/.

[14] R. Yerra and Y.-K. Ng. Detecting similar html documents using a fuzzy set information retrieval approach. In Processings of IEEE International Conference on Granular Computing ( $\left.G r C^{\prime} 05\right)$, pages 693-699, 2005.

[15] L. Zadeh. Similarity relations and fuzzy orderings. Information Sciences, 3:177-200, 1970.

[16] Y. Zhao and G. Karypis. Topic-driven clustering for document datasets. In Processings of SIAM International Conference on Data Mining, pages 358-369, 2005. 